\title{
Images in and of the brain
}

\section{It is beginning to be possible to produce images of the brain in action, but interpretation of the pictures obtained remains uncertain.}

DRAMATIC pictures of the brain in action are now being obtained by optical recording using voltage-sensitive dyes and by positron emission tomography. Some of them have been published in Nature (Blasdel, G.G. \& Salama, G. 321, 579; 1986 and Fox, P.T. et al. 323, 806; 1986); last week, A. Grinvald et al. (Nature 324, $361 ; 1986)$ reported an optical method for recording cortical activity without the use of dyes, at least when the surface of the cortex can be made accessible.

Producing these images requires sophisticated computer processing; understanding them needs tutored interpretation, involving some of the same neural mechanisms that are the subject of the images. Problems such as these were discussed at a recent symposium* on images and understanding, which brought together people who generate images, from the arts and computer graphics, together with neuroscientists and artificial intelligence people who seek to understand the mechanisms by which the brain analyses images.

The conundrum of how the brain interprets images of the brain was illustrated by Horace Barlow (Cambridge) and Marcus Raichle (St Louis), but the implications were left unexplored. The advantage of the new cortical imaging techniques is that the activity of many neurons can be monitored at once, in the intact brain, so as to give a much more complete picture of the spatial organization of neuronal responses to particular stimuli than could previously be had by painstaking reconstruction, and, with some guesswork, from single-unit recordings. Microelectrode recording is, of course, not superseded by the glamorous new technology, for it remains the best way of characterizing the responses of individual neurons. Rather, the synoptic methods add a new dimension by making it possible to see all the neurons activated at once by a particular stimulus; they provide functional maps of the brain.

What do such maps reveal? It has long been known that the brain itself contains a map of the external world - in several areas of the cortex, there are detailed point-to-point, though not necessarily proportional, representations of sensory fields or of the body surface. A consistent finding is that the world is represented many times over, both within a single area and in several regions; different aspects of

-International Symposium on Images and Understanding. Royal Society, London, 6-8 October I986. the same information are then processed in parallel channels.

Colin Blakemore (Oxford) suggested that one reason for such maps is to bring common functions to adjacent points for efficient processing of information. This may be true for orientation and ocular dominance (the basis of depth perception) in the striate area of the visual cortex, as Blasdel and Salama's voltage-sensitive dye maps suggest. But in the same region, cells responding to colour and direction of movement are physically intermingled yet functionally quite separate and they project to separate secondary visual areas (Hubel, D.H. \& Livingstone, M.S. Nature 315, 325; Shipp, S. \& Zeki, S. Nature 315, 322; DeYoe, E.A. \& Van Essen, D.C. $317,58 ; 1985)$. The organization at these relatively low levels in the processing hierarchy is probably a sorting process through which the correct combinations become addressed to multimodal processing centres higher up the system.

All the maps of the world are symbolic, the maps of the brain included. Reading them must be learned, just as people must learn to interpret geographical maps. Because it is still early in the development of the new imaging technology, the symbolic conventions are not well established and the viewer has to work hard to translate grey-scale or false-colour images into patterns of cortical activity. What is more, the published images are static snapshots of activity patterns at different times.

One of the most difficult features of the brain to capture and analyse is the pattern of dynamic change in the activity of and interaction between its constituent neurons. Because animals and the world around them are rarely still, the sensory input to the brain is constantly changing. Even when one looks at a static image, the attention shifts to focus on different aspects - and appreciation of the same image on different occasions is tempered by physiological and psychological states.

Others know of the difficulty. The art historian, Ernst Gombrich, pointed out how difficult it is to represent a sequence of movements pictorially without recourse to symbols or verbal explanation. Pictorial instructions are therefore rarely universally comprehensible. A clear example of how the interpretation of symbols depends on cultural context and hence on learning was provided by Monica Parker, choreologist to the Royal Ballet, London. In the Benesh notation for recording movements, a basic diagram representing a simple position with arms outstretched at shoulder height would look very different if adopted by an Indian classical dancer rather than by a Western dancer. The details that give the position its character will depend on the form of expression inherent in the tradition of the interpreter.

Although many of the visual images we create and enjoy are static, the perception of movement is a basic and important aspect of visual analysis. In primates, the motion-processing channel starts in the retina but directional sensitivity is first displayed by neurons in area V1 of visual cortex and further elaborated in the motion-analysing area, called MT.

Neurons in MT project to association areas including temporal cortex where, in monkeys, David Perrett and colleagues at St Andrews have recorded from many visually driven cells selective for aspects of body movements. Their results begin to give a glimpse of how the brain reconstructs complex images. Each cell responds to limited views of the body and limited sets of movements, for instance, to a body facing and moving towards the viewer or to a particular articulation of a limb. Many do not depend on absolute form, but respond similarly to a monkey, a human being or with a pattern of dots, as long as it is moving correctly. Although these higher-order cells combine information about motion, form, colour and depth, each still responds only to selected features of the object. Specific recognition or perception of the body as a whole must be the product of collaboration between many cells. Where and how this occurs for any image, simple or complex, is one of the unsolved mysteries of neuroscience.

As a highly visual species, how human beings perceive the world is bound to be a challenging subject; most research on sensory processing and perception has focused on the visual system. But it was for an artist, Liliane Lijn, to point out that ultimately our perception of the world is multisensory; visual images are reinforced by sound, smell and touch. How and where in the brain all the parallel channels carrying the various aspects of all the stimuli converge are unanswered questions. Beyond that still are our conceptual capabilities, defined by Richard Gregory (Bristol) as the marshalling of ideas on a long timescale to develop longterm strategies, generalizations and abstractions. 